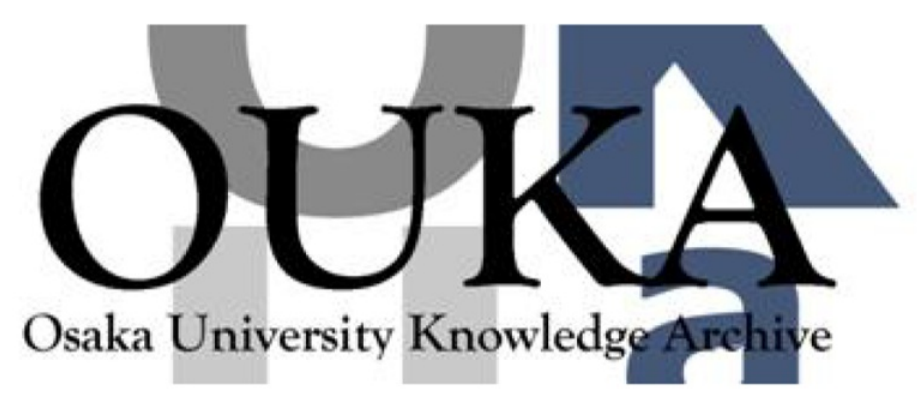

\begin{tabular}{|c|l|}
\hline Title & $\begin{array}{l}\text { Laser actions in nano-helical-structured liquid } \\
\text { crystals }\end{array}$ \\
\hline Author(s) & $\begin{array}{l}\text { Ozaki, Masanori; Ozaki, Ryotaro; Matsui, } \\
\text { Tatsunosuke et al. }\end{array}$ \\
\hline Citation & $\begin{array}{l}\text { Proceedings of SPIE - The International Society } \\
\text { for Optical Eng ineering. 5518 p. 44-p.50 }\end{array}$ \\
\hline Issue Date & $2004-10-15$ \\
\hline oaire:version VoR \\
\hline URL & https://hdl. handle.net/11094/76950 \\
\hline rights & \\
\hline Note & \\
\hline
\end{tabular}

Osaka University Knowledge Archive : OUKA

https://ir. Library. osaka-u. ac. jp/

Osaka University 


\title{
Laser actions in nano-helical-structured liquid crystals
}

\author{
Masanori Ozaki*, Ryotaro Ozaki, Tatsunosuke Matsui, and Katsumi Yoshino \\ Department of Electronic Engineering, Graduate School of Engineering, Osaka University \\ Yamada-Oka, Suita, Osaka 565-0871 Japan
}

\begin{abstract}
Optically pumped laser action has been observed at the edge wavelength of the stop band in a dye-doped flexible freestanding film of photopolymerized cholesteric liquid crystal (PCLC), which originates from band edge effect of the onedimensional photonic band gap. On the other hand, defect mode laser action has also been experimentally demonstrated at the defect mode wavelength within the band gap. This laser action is based on the photon localization at the twist defect of the composite film consisting of two PCLC layers. Twist defect mode (TDM) is induced by the introduction of twist defect which is a discontinuity of the director rotation around the helix axis at an interface of two PCLC layers. We also propose a new type of tunable defect mode based on the chiral defect in which the partial modulation of the helix pitch is introduced.
\end{abstract}

Keywords: photonic crystal, liquid crystal, laser action, helical structure, cholesteric liquid crystal, defect mode

\section{INTRODUCTION}

Liquid crystals including chiral molecule have a self-organized helical structure which can be regarded as a onedimensional (1-D) periodic structure. In such systems, there is a so-called stop band in which the light can not propagate, which is considered as a 1-D pseudo-bandgap. In a 1-D periodic structure, the laser action has been expected at the photonic band edge where the photon group velocity approaches zero. ${ }^{1}$ Lasing at the band edge has been reported in the cholesteric liquid crystal (CLC), ${ }^{2.3}$ chiral smectic liquid crystal ${ }^{4-6}$, cholesteric blue phase ${ }^{7}$ and polymerized cholesteric liquid crystal..$^{8 \cdot 10}$

On the other hand, the localization of the light based on the defect mode caused by the imperfection in periodic structure has been expected as potential applications such as low threshold lasers and micro waveguides. ${ }^{11.15}$ The introduction of the defect layer into the periodic helical structure of the CLCs has been theoretically studied. ${ }^{16,17}$ Especially, Kopp et al. have predicted the existence of a localized state for single circularly polarized light in the twist defect of the CLCs. ${ }^{17}$ However, in spite of the theoretical demonstration of the twist defect mode (TDM) in the CLC, evident experimental demonstration on such defect mode has not been carried out.

In this study, we experimentally demonstrate the defect mode in the 1-D photonic band gap of the CLC film having a twist defect. The laser action based on the TDM is also observed in the dye-doped photopolymerized CLC (PCLC) composite film with the twist defect. ${ }^{18}$ Although TDM has a high quality (Q) factor, its frequency can not be tuned by an external field in this system. We propose a new type of defect mode based on the chiral defect in which the partial modulation of the helix pitch is induced. In the new chiral defect mode, the tuning of the mode frequency can be expected upon partially modulating the helical twisting power.

\section{EXPERIMENTAL}

For the fabrication of PCLC, two types of photo-polymerizable CLC mixtures (Merck KGaA) were used, which have right-handed helix and reflection bands around $779 \mathrm{~nm}$ and $440 \mathrm{~nm}$. The helical pitch of the CLC sample, that is, the wavelength of the optical stop band can be adjusted by mixing these two CLC compounds at a proper ratio. The freestanding CLC polymer film was prepared as follows. The monomer sample was inserted by a capillary action into the sandwiched cell that is composed of two grass plates. In order to obtain a homogeneously aligned cell, the surfaces were 
coated with a polyimide (Japan Synthetic Rubber, AL1254) and rubbed. The cell gap was $25 \mu \mathrm{m}$. The. CLCs in this cell align their director parallel to the glass plates, that is, the helical axis is perpendicular to the glass substrates. UV light irradiation was performed using a Xe lamp to induce the photo-polymerization of the UV-curable CLC monomer. After UV light irradiation, two glass substrates were removed and the free-standing PCLC film was obtained. As a laser dye dopant in the CLC, [2-[2-4-(Dimethylamino)phenyl]ethenyl]-6-methyl-4H-pyran-4-ylidene] propanedinitrile, DCM (Exciton) was used. The concentration of the dye was 0.4 wt.-\%.

The PCLC film with the twist defect was prepared as follows. The photo-polymerizable CLC monomer was spin-coated from a toluene solution on a glass substrate on which a polyimide (AL-1254) was coated and rubbed in one direction. In order to obtain a uniform planer alignment, the coated CLC was annealed at the temperature just below a clearing point. The CLC on the substrate aligns their director parallel to the glass plate, that is, the helical axis is perpendicular to the glass substrate. UV light irradiation was performed using a Xe lamp to induce the photo-polymerization of the UVcurable CLC monomer. Two PCLC films were put together as the directors of liquid crystal molecules at an interface of these films make a certain angle $\phi$ as shown in Fig.1. In other words, there is a discontinuous phase jump of the azimuthal angle of the helical structures between these PCLC films at the interface, which acts as a twist defect in the helicoidal periodic structure. For the lasing experiment, [2-[2-4-(Dimethylamino) phenyl] ethenyl]-6-methyl- 4H-pyran4ylidene] propanedinitrile, DCM (Exciton) was doped in the PCLC. The concentration of the dye was 0.4 wt.- $\%$.
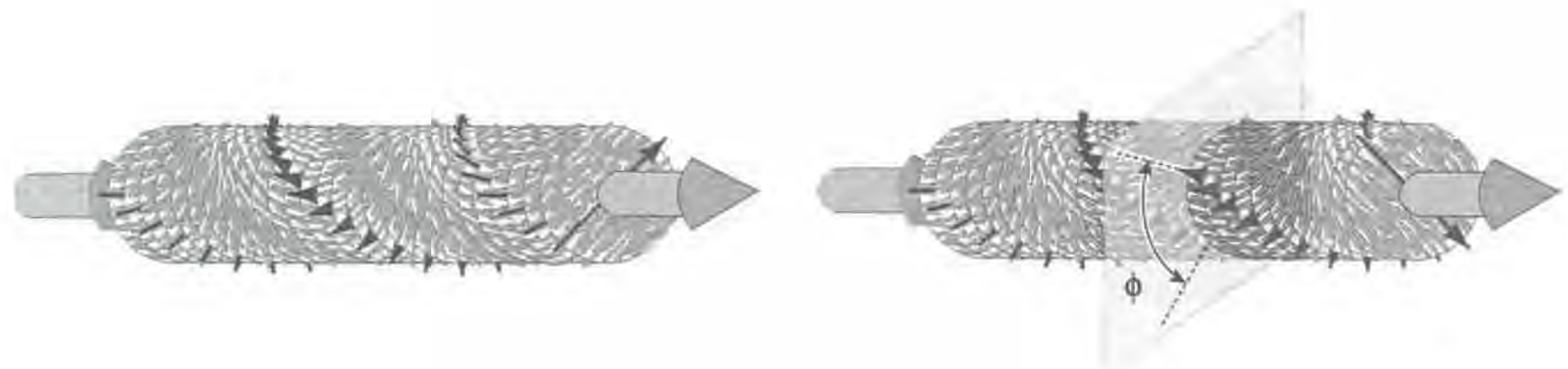

Figure 1: Schematic explanation of the double PCLC composite film having a twist defect which is a discontinuity of the director rotation around the helix axis.

\section{RESULTS AND DISCUSSION}

\subsection{Laser action in photopolymerized CLC}

The emission spectrum of the dye-doped PCLC film for excitation energy of $30 \mu \mathrm{J} /$ pulse is shown in Fig.2. A second harmonic light of Q-switched Nd:YAG laser was used for an excitation light source. The illumination area on the sample was about $0.2 \mathrm{~mm}^{2}$. When the excitation energy is low $(<3.0 \mu \mathrm{J} / \mathrm{pulse})$, the spectrum is dominated by a broad spontaneous emission. At high excitation energy, laser action is confirmed at the edge of the stop band which is observed as a suppression of the transmittance. The full width at half maximum (FWHM) of the emission peak is about $2 \mathrm{~nm}$, which is limited by the spectral resolution of our spectrograph. At lower excitation energies, the emission intensity increases in proportion to the pump energy, while above the threshold, the emission intensity increases steeply. The linewidth of the emission spectrum also drastically decreases above the threshold. This laser action is achieved without any substrates and is observed even when PCDL film is bent. This suggests that 1-D helical structure required for the laser action is maintained even in the deformed film. This flexibility may enable us to fabricate optical devices with new functionalities. 


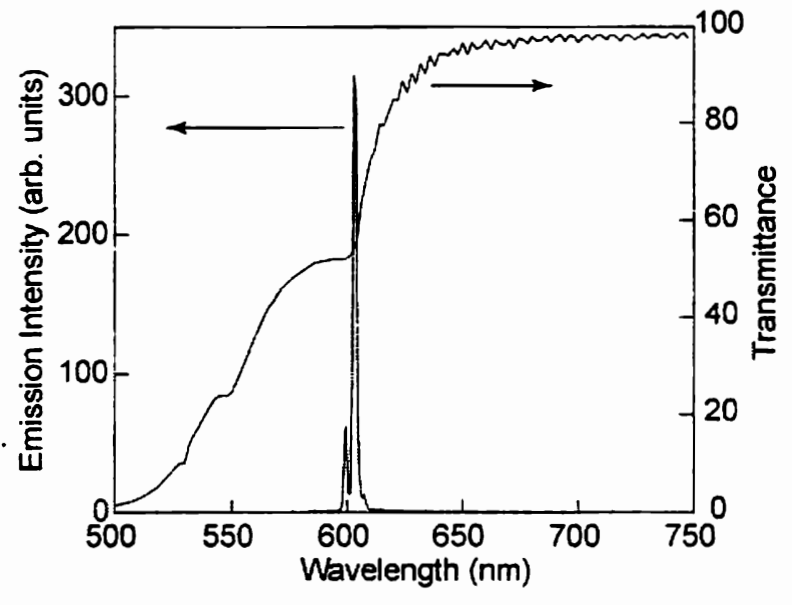

Figure 2: Emission and transmission spectra of dyedoped PCLC film.

\subsection{Twist defect mode lasing}

Laser actions reported so far in chiral liquid crystals are observed at the edge wavelength of the stop band and are associated with the group velocity anomaly at the photonic band edge. On the other hand. low threshold laser action based on the photon localization at the defect in periodic structure can also be expected. The introduction of the defect into the periodic helical structure of the CLCs has been theoretically studied. Especially, Kopp et al. have predicted the existence of a single circularly polarized localized mode in the twist defect of the CLCs. ${ }^{17}$

Figure 3(a) shows the transmission spectrum of the dyedoped double PCLC composite film containing a discontinuous defect interface. A stop band, 1-D photonic band gap, is confirmed in the spectral range from $580 \mathrm{~nm}$ to $640 \mathrm{~nm}$. It should be noted that a sharp peak appears at 611 $\mathrm{nm}$ within the photonic band gap. which might be related to the defect mode induced by the introduction of the twist defect interface.

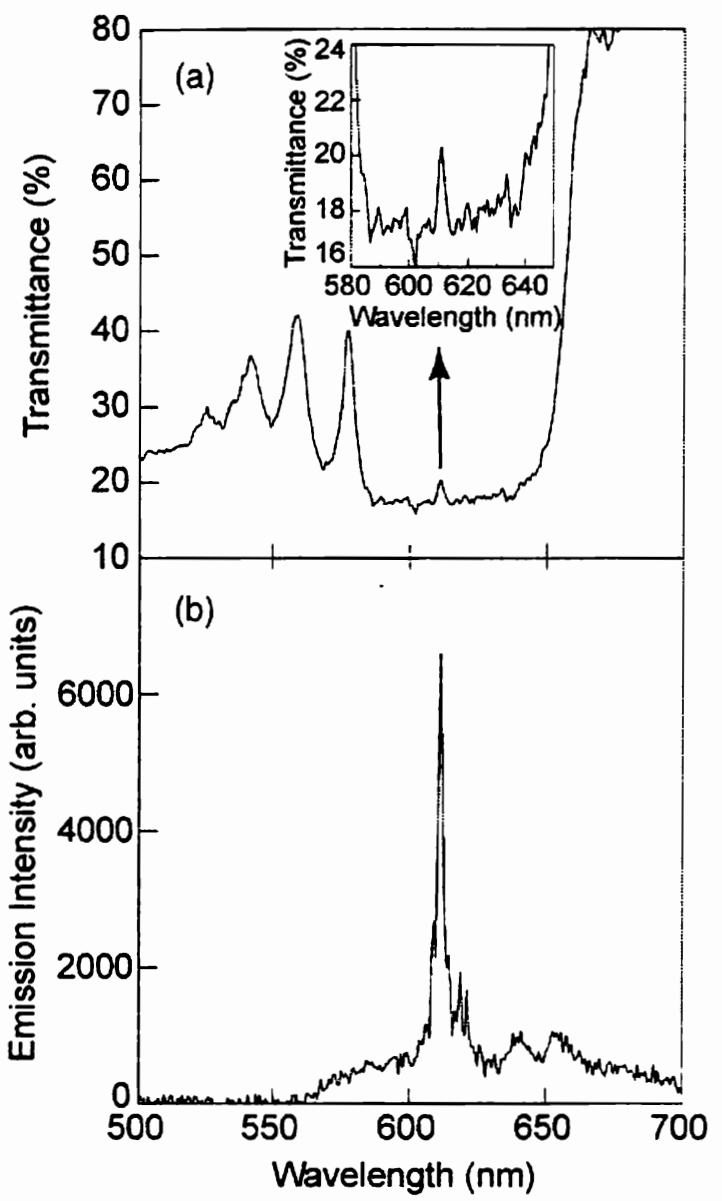

Figure 3: (a) Transmission spectrum of the dyedoped double PCLC composite film with twist defect. (b) Emission spectrum of the double PCLC composite film at above the threshold pump pulse energy ( $200 \mathrm{~nJ} /$ pulse).

We have performed theoretical calculation of light propagation in PCLC films with and without twist defect interface, using a method of characteristic matrices. This method is a numerical analysis based on the Maxwell equation which can be used to quantitatively calculate the light propagation in the medium with refractive index varying along one direction. Assuming that the phase jump of the director angles $\phi$ at the interface of two PCLC layers is $4 \pi / 9$ rad, the theoretically calculated results are in good agreement with the experimental ones. This result indicates that the sharp peak observed in the photonic band gap corresponds to the TDM in the double PCLC composite film with the twist defect interface.

Figure 4 shows the electric field distribution of the propagating light in the PCLC film with twist defect, evaluated using a finit difference time domain (FDTD) calculation. At the twist defect interface, there is a phase jump of the director rotation around the helix axis. The light propagates from the right side to the left. The light intensity is localized at the defect interface, resulting in a partial transmission of the light. 


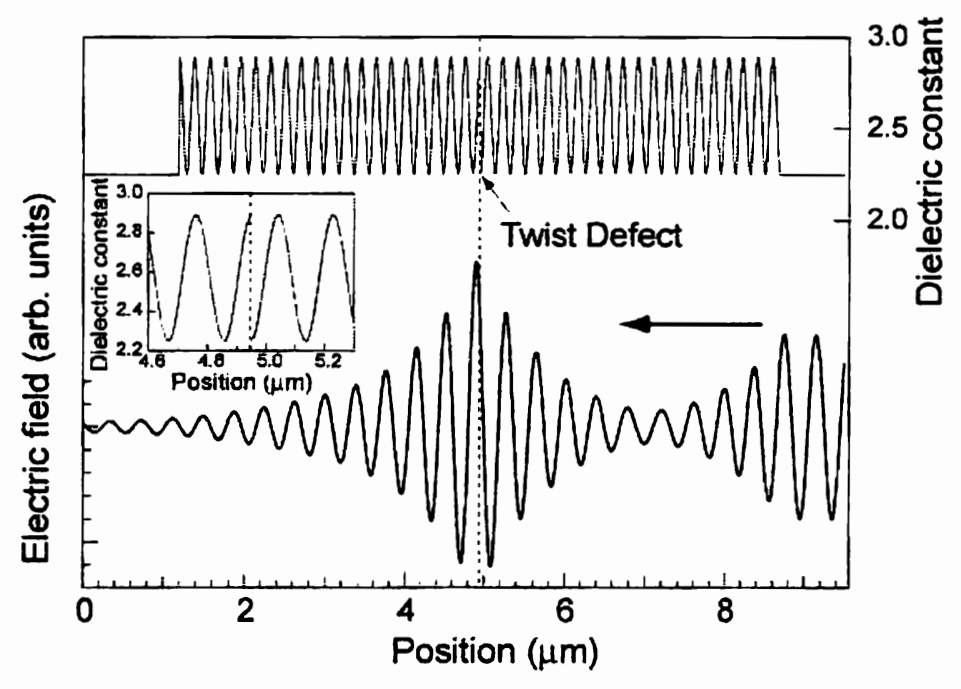

Figure 4: Electric field distribution of the propagating light in the PCLC with twist defect.

Figure 3 (b) shows the emission spectrum of the dye-doped double PCLC composite film with the defect interface at pump energy of 200nJ/pulse. For an excitation source, second harmonic light of a mode-locked Nd:YAG laser (Ekspla, PL2201) was used. The pulse width, wavelength and pulse repetition frequency of the pump laser beam were 100ps, $532 \mathrm{~nm}$ and $1 \mathrm{kHz}$, respectively. The illumination area on the sample was about $0.2 \mathrm{~mm}^{2}$. For low excitation energy, the spectrum is dominated by a broad spontaneous emission. At high excitation energy ( $200 \mathrm{~nJ} / \mathrm{pulse}$ ), however. laser action appears at $611 \mathrm{~nm}$ which is within the band gap and coincides with the TDM wavelength. The FWHM of the emission peak is about $2 \mathrm{~nm}$, which is limited by the spectral resolution of our experimental setup. Figure 5(a) shows the peak intensity of the emission at $611 \mathrm{~nm}$ as a function of excitation energy. Above the threshold at a pump pulse energy of about 100nJ/pulse, the emission intensity increases. The FWHM of the emission spectrum also drastically decreases above the threshold. These results confirm that laser action occurs above the threshold of the pump energy at the wavelength of the TDM in the photonic band gap.
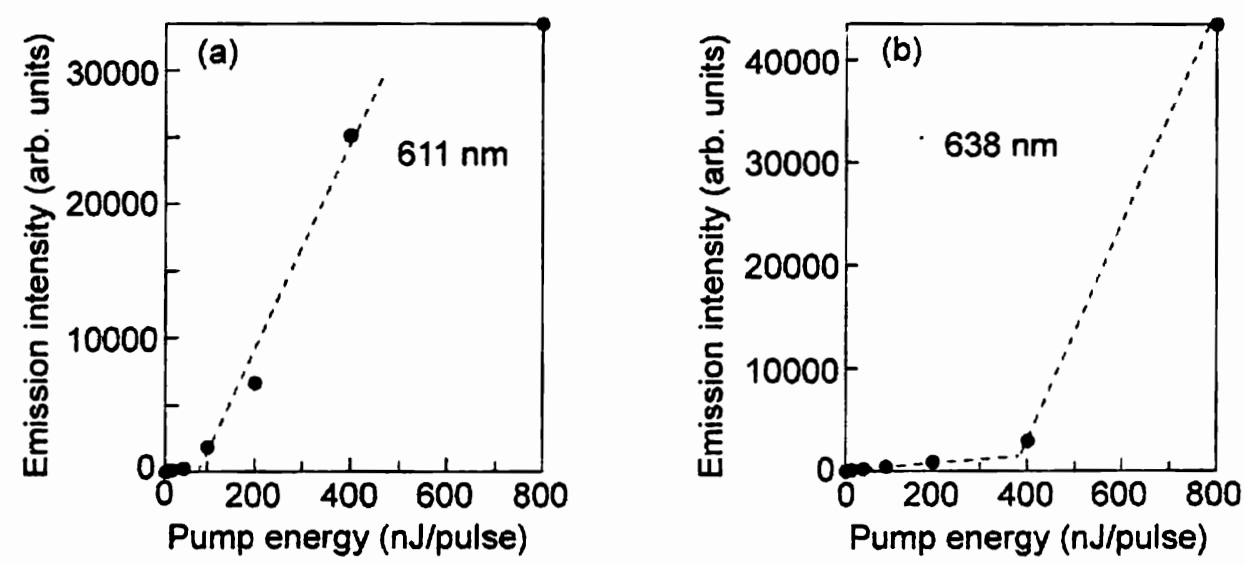

Figure 5: Pump energy dependences of the intensity of emission peaks at $611 \mathrm{~nm}$ and $638 \mathrm{~nm}$ of dye-doped PCLC with twist defect. 


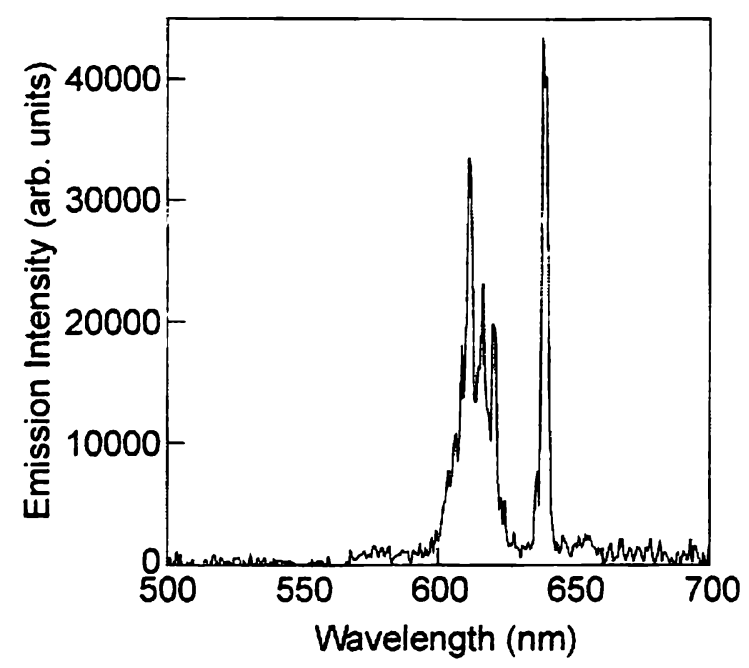

Figure 6: Emission spectrum of dye-doped PCLC with twist defect at high pumping energy of $800 \mathrm{~nJ} /$ pulse.

With increasing excitation energy more, another emission peak grows up. Figure 6 shows emission spectrum of dyedoped PCLC with twist defect at excitation energy of 800nJ/pulse. Besides the peak due to TDM $(611 \mathrm{~nm})$. another sharp emission peak appears at $638 \mathrm{~nm}$ which corresponds to the edge wavelength of the stop band. Figure 5 (b) shows excitation energy dependence of the emission peak intensity at $638 \mathrm{~nm}$. Above the threshold excitation energy (400nJ/pulse), emission intensity drastically increases. This emission peak might be associated with the band edge lasing which appears in PCLC without defect as shown in Fig.2. Consequently, the defect mode lasing occurs at lower pumping energy compared with that of band edge lasing, while at high excitation energy the defect mode lasing reaches the limit and band edge lasing appears.

\subsection{Transient defect mode induced by the partial deformation of helix}

TDM based on the composite film of two PCLCs has been achieved. However, its wavelength can not be tuned by an external field such as the electric field or light. We have proposed a new type of defect mode in the helix which can be dynamically tuned by the external field. ${ }^{19}$ Figure 7 shows schematic explanation of a photonic defect in CLC. If the periodicity (pitch) of the helix is partially changed. that is, the pitch is partially squeezed or expanded, these irregularities in the periodic structure should act as a defect and cause the light localization. As a method to induce partial change in helix pitch, we suppose that the local modification of HTP is induced by a focused Gaussian laser light. Optical control of HTP can be realized using photochemical effects of the doped azobenzene, nonlinear optical effects, or by simple heating. Photoinduced reversible control of HTP of CLC has been demonstrated in the CLC containing photochromic azobenzene, and applications to a reflection-type display devices. an optical shutter, an optical memory, and so on have been studied. ${ }^{20.21}$ By the trans-cis photoisomerization of the doped azobenzene. HTP of the host CLC changes, so that photoinduced control of HTP can be realized. On the other hand, Winful theoretically suggested that an intense light can induce a change of HTP of the CLC by the coupling of the optical field to the local dielectric anisotropy. ${ }^{22}$ Moreover, the helical pitch of the CLC depends on temperature, so that a local deformation of helix can also be performed by an optical heating. From these characteristics of the CLC and the mixture with azobenzene molecules, our model of calculation can be considered as a realizable assumption. 

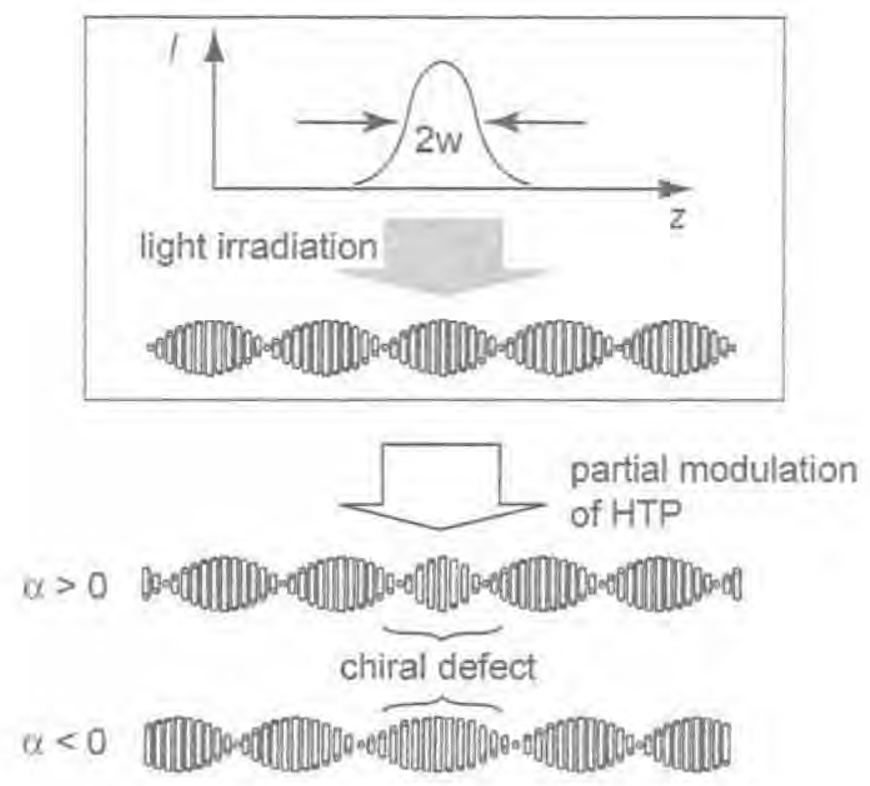

Figure 7: Schematic explanation of the optically induced chiral defect based on the partial deformation of the helix pitch.

In order to check the validity of this model, we carried out a simple simulation using Berreman's $4 \times 4$ transfer matrix. ${ }^{23}$ First, let me consider when the helix is illuminated by a Gaussian beam light with a following intensity distribution, $I(z)=I_{0} \exp \left(-2 z^{2} / w^{2}\right)$. Here, $z$ axis is parallel to the helix axis and $2 w$ is a beam diameter of the light. Assuming that the induced modulation of HTP is proportional to the intensity of the writing light $I(z)$, the distribution of the HTP, $\operatorname{HTP}(z)$, can be expressed as

$$
H T P(z)=H T P_{0}\left\{1+\alpha \exp \left(-2 z^{2} / w^{2}\right)\right\}
$$

where $\alpha$ is a modulation strength of HTP and $H T P_{0}$ is an initial HTP. The value of $\alpha$ depends on the concentration of dopants, the intensity of the light. absorption of the doped dye, and so on. which can be controlled by the external field.

Figure 8 shows the transmission spectra for the right-handed circularly polarized (RCP) and left-handed circularly polarized (LCP) lights passing through the CLC having the chiral defect induced partial helix deformation $(\alpha=-0.2, w=300 \mathrm{~nm})$. The helix sense of CLC is right-handed. The pitch of the helix is $350 \mathrm{~nm}$, and ordinary and extraordinary refractive indices of LC are 1.5 and 1.7, respectively. The thickness of the CLC is $5 \mu \mathrm{m}$.

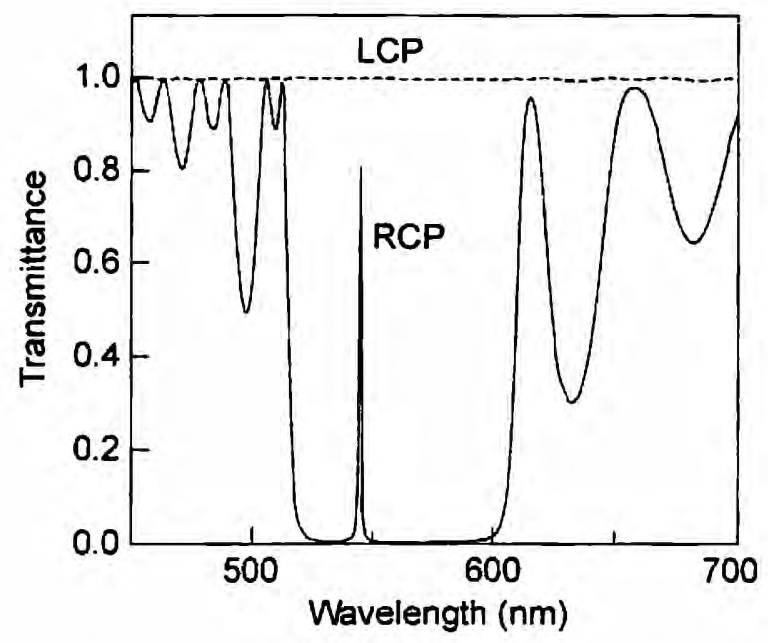

Figure 8: Transmission spectra of RCP and LCP lights passing through the CLC with deformation-induced chiral defect.

A transmission peak based on the defect mode was observed in the stop band for the RCP light. On the other hand, when the incident light was LCP. no defect mode was observed. In other words, the defect mode due to the photon localization 
in our system can be realized only for the circularly polarized light with the same handedness as the helix, which is similar to the result of TDM. The position of the defect mode depends on $\alpha$ and $w$. With decreasing $\alpha$, a transmission peak due to the defect mode shifted toward the longer-wavelength edge. On the contrary, with increasing $\alpha$, peak shifted toward the shorter-wavelength edge. The increase and decrease in $\alpha$ correspond to the squeeze and expansion of the helix pitch. Therefore, it was found that the tuning of defect modes could be achieved by partial squeeze and expansion of the helix.

\section{SUMMARY}

Two types of laser actions were demonstrated using PCLC. They originated from the band edge effect of the onedimensional photonic bands gap and the defect mode within the band gap. We experimentally demonstrated the TDM in the 1-D photonic band gap of the CLC film having a twist defect which was a discontinuity of the director rotation around the helix axis at an interface of two PCLC layers. The laser action based on the TDM was also observed in the dye-doped PCLC composite film with the twist interface. We proposed a new type of defect mode based on the chiral defect in which the partial modulation of the helix pitch was induced. In the new chiral defect mode, the tuning of the mode frequency could be expected by partial modulation of the helical twisting power.

This work is supported by a Grant-in-Aid for Scientific Research from the Japan Ministry of Education, Culture. Sports, Science and Technology (15656083). We would like to show our acknowledgements to Merck KGaA for providing the photo-polymerizable CLC materials.

\section{REFERENCES}

1. J. P. Dowling. M. Scalora, M. J. Bloemer, C. M. Bowden, J. Appl. Phys. 75, 1896, 1994.

2. V. I. Kopp, B. Fan. H. K. Vithana, A. Z. Genack, Opt. Lett. 23. 1707, 1998.

3. B. Taheri, A. F. Munoz, P. Palffy-Muhoray, R. Twieg, Mol. Cryst. Liq. Cryst. 358, 73, 2001.

4. M. Ozaki. M. Kasano, D. Ganzke, W. Haase, K. Yoshino, Adv. Mater. 14. 306, 2002.

5. M. Ozaki, M. Kasano, D. Ganzke, W. Haase, K. Yoshino, Adv. Mater. 15. 974, 2003.

6. M. Kasano, M. Ozaki. D. Ganzke, W. Haase, K. Yoshino, Appl. Phys. Lett., 82. 4026, 2003.

7. W. Cao, A. Munoz. P. Palffy-Muhoray, B. Taheri, Nature Mater. 1, 111, 2002.

8. H. Finkelmann, S. T. Kim, A. Munoz, P. Palffy-Muhoray, B. Taheri, Adv. Mater. 13, 1069, 2001.

9. J. Schmidtke. W. Stille. H. Finkelmann, S.T. Kim, Adv. Mater. 14, 746, 2002.

10. T. Matsui, R. Ozaki, K. Funamoto, M. Ozaki, K. Yoshino, Appl. Phys. Lett., 81, 3741, 2002.

11. J. S. Foresi. P. R. Villeneuve, J. Ferrera, E. R. Thoen, G. Steinmeyer, S. Fan, J. D. Joannopoulos, L. C. Kimerling, H. I. Smith, E. P. Ippen, Nature 390, 143, 1997.

12. O. Painter. R.K. Lee, A. Scherer, A. Yariv, J.D. O’Brien, P.D. Dapkus, I. Kim, Science 284. 1819, 1999.

13. S. Noda, A. Chutinan. M. Imada, Nature 407, 608, 2000.

14. T. Hattori, N. Tsurumachi, H. Nakatsuka, J. Opt. Soc. Am. 14. 348, 1997.

15. R. Ozaki. T. Matsui. M. Ozaki, K. Yoshino, Jpn. J. Appl. Phys. 41, L1482, 2002.

16. Y. C. Yang. C. S. Kee, J. E. Kim. H. Y. Park, J. C. Lee, Y. J. Jeon, Phys. Rev. E 60, 6852, 1999.

17. V. I. Kopp, A. Z. Genack, Phys. Rev. Lett. 89, 033901, 2002.

18. M. Ozaki, R. Ozaki. T. Matsui, K. Yoshino, Jpn.J.Appl.Phys., 42, L472, 2003.

19. T. Matsui, M. Ozaki, K. Yoshino, Phys. Rev. E, 69, 061715, 2004.

20. E. Sackmann, J. Am. Chem. Soc. 93. 7088, 1971.

21. H. K. Lee, K. Doi, H. Harada, O. Tsutsumi, A. Kanazawa. T. Shiono, T. Ikeda, J. Phys. Chem. B 104, 7023, 2000.

22. H. G. Winful, Phys. Rev. Lett. 49, 1179, 1982.

23. D. W. Berreman, J. Opt. Soc. Am. 62, 502, 1972. 